\title{
Does an Electronic Health Record Improve Completeness of Prenatal Studies?
}

T.A. McLean'; A.K. Lewkowitz²; E. Test²; M.G. Zlatnik²

${ }^{1}$ Kaiser Permanente, Obstetrics and Gynecology, Livermore, CA, United States; ${ }^{2}$ UCSF, Obstetrics, Gynecology, \& Reproductive Sciences, San Francisco, CA, United States

\section{Keywords}

Electronic health record, prenatal care

\section{Summary}

Objective: To determine whether implementation of an electronic health record (EHR) would increase the rate of prenatal Human Immunodeficiency Virus (HIV) and purified protein derivative (PPD) testing.

Methods: Eligible participants received prenatal care and delivered at term at a single academic institution in March-April 2011, March-April 2012, and March-April 2013. As part of routine prenatal care, all women were tested for HIV and tuberculosis (via a PPD test) during each pregnancy. The 2011 cohort was charted on paper. The 2012 and 2013 cohorts were charted via EHR. To appear in the prenatal labs display in EHR, PPD results must be manually documented, while HIV results are uploaded automatically. Documentation of PPD and HIV tests were analyzed.

Results: The 2011, 2012, and 2013 cohorts had 249, 208, and 190 patients, respectively. Complete PPD and HIV results were less likely to be charted in the 2012 EHR cohort compared to the paper chart cohort $(72.1 \%$ vs. $80.1 \% ; p=0.03)$. This was driven by fewer documented completed PPD tests ( $201183.9 \%$ vs. $201272.6 \%$; $p=0.003$ ). PPD test documentation improved non-significantly to $86.2 \%$ in the 2013 EHR cohort $(p=0.5)$. HIV documentation rates increased from $95.2 \%$ in the paper chart cohort to $98.6 \%$ in the 2012 EHR cohort $(p=0.04)$, and to $98.9 \%$ in the 2013 EHR cohort $(\mathrm{p}=0.03)$.

Conclusions: EHR implementation corresponded with a marked decrease in documentation of PPD test completion. HIV documentation rates improved. PPD results were likely charted incorrectly in provider notes due to training deficiencies and lack of standardization, which did not improve significantly after retraining.

Correspondence to:

Tracey McLean

Department of Obstetrics and Gynecology

The Permanente Medical Group

Livermore, $\mathrm{CA}$

Email: tracey.a.mclean@kp.org
Appl Clin Inform 2015; 6: 669-676

http://dx.doi.org/10.4338/ACl-2015-05-RA-0062

received: May 16, 2015

accepted in revised form: September 21, 2015

published: November 11, 2015

Citation: McLean T, Lewkowitz A, Test E, Zlatnik M.

Does an electronic health record improve completness of prenatal studies? Appl Clin Inform 2015; 6:

669-676

http://dx.doi.org/10.4338/ACI-2015-05-RA-0062 


\section{Introduction}

In 2009, the Health Information Technology for Economic and Clinical Health (HITECH) Act was enacted, allocating nearly $\$ 19$ billion towards expanding the adoption of electronic health records (EHRs) in the American health-care system [1]. Nationwide implementation of EHRs is intended to lower administrative costs, streamline patient care, improve surveillance, and increase efficiency [1, 2]. Indeed, data suggest that, compared to paper charting, EHRs can increase the quality, safety, and efficiency of patient care by improving the legibility and comprehensiveness of, and access to, patient notes, implementing standardized patient quality measures, and streamlining clinical processes [2-7]. However, the effectiveness of EHRs may be limited by cost, by the need for all medical personnel be trained on correct EHR usage, and by the inability of different EHR systems to share medical information about the same patient [8].

Pregnancy is an ideal condition to evaluate whether the EHR's potential outweighs its limitations: comprehensive prenatal care includes standardized testing in both outpatient and inpatient settings that guides multiple medical providers' management of antepartum, intrapartum, and postpartum care [9]. Thus, prenatal care demands an EHR that concisely transfers information between different medical settings, helps ensure prenatal care testing is completed according to standardized guidelines, and is easily accessible by and understandable to various medical personnel. Though limited, data on EHRs in the prenatal setting is positive: studies suggest that EHRs improve communication between prenatal care networks [10], increase both timeliness of prenatal laboratory testing [2] and completeness of the prenatal record [5], and decrease the rate of newborn interventions and unnecessary maternal testing [11].

Analysis of prenatal laboratory results is an interesting area of evaluation because some results, such as infectious disease antibody testing, can be automatically uploaded into the EHR. Other results, such as those of the purified protein derivative (PPD) test for tuberculosis, point of care tests, and immunization dates, must be manually entered into the correct section of the EHR to propagate correctly within the prenatal care record. Additionally, the EHR can be designed to offer alerts or visual cues to providers when results are lacking.

\section{Objectives}

This study compares prenatal test completion pre- and post-EHR implementation, by determining how EHR implementation impacts documentation rates of prenatal testing for human immunodeficiency virus (HIV) and tuberculosis. These tests are ordered routinely during every pregnancy at our institution. New prenatal HIV diagnoses are not common but have urgent clinical implications for the pregnancy and delivery. The incidence of tuberculosis in our community is significantly higher than average. For example, in 2014 the incidence of new cases was nearly four times the national average [12]. While the absolute number of cases diagnosed prenatally is low, this testing is part of a public health effort to identify patients with latent infections who may not interact with the health system outside of pregnancy. These tests were chosen for study because they represent both types of resulting mechanisms: HIV results propagate automatically into the EHR, while PPD test results must be manually entered. We hypothesized that test completion, and thus documentation in the prenatal console, would increase with EHR implementation because the prominently displayed prenatal console would render all missing prenatal tests more visible to all prenatal providers.

\section{Methods}

This study was conducted at an urban academic medical center. Prior to late 2011, we utilized paper charts for all ambulatory prenatal care: prenatal testing results were manually written into each patient's paper chart, which was stored in the outpatient clinic. The chart was brought to labor and delivery during a patient's admission, where a legacy electronic note charting system was in place. A comprehensive, enterprise-wide EHR was launched in the ambulatory prenatal clinics in July 2011 and on labor and delivery in June 2012. Providers underwent the EHR manufacturer's recom- 
mended training, consisting of eight hours of classroom instruction. The EHR interface includes a prenatal console, in which designated prenatal laboratory results are displayed prominently. Results that come from the laboratory, such as for HIV, display automatically. PPD test results must be manually inputted. Results from the console are propagated automatically in labor and delivery's admission history and physical.

This retrospective cohort study was approved by the Institutional Review Board and included women with singleton pregnancies who received their prenatal care and delivered at term at our institution. Preterm deliveries were excluded from our cohort to eliminate a potential confounder of HIV or PPD test non-completion-unplanned premature delivery-from our analysis. Of note, all women in our prenatal clinics receive HIV testing with first- and third-trimester laboratory testing as well as PPD testing at their convenience during each pregnancy.

Women were stratified into three cohorts according to the year of their delivery. The first cohort delivered in March and April 2011, prior to implementation of the EHR, when paper prenatal charts were utilized. The second cohort delivered in March and April of 2012 and had their entire prenatal record documented in the ambulatory EHR. The third cohort delivered in March and April of 2013. Completion rates of HIV and PPD tests were assessed via a chart review, performed by three physicians. Data were also collected on parity and insurance type (public or private, as a proxy for socioeconomic status) to determine whether these factors affected test completion rates. Of note, preliminary results from the first two cohorts of the study were presented in an intradepartmental meeting to all prenatal providers, prompting the creation of a brief EHR refresher course.

Based on our prior clinical experience, we estimated that approximately $85 \%$ of women had both HIV and PPD documented in their chart prior to implementation of the EHR. We hypothesized that this would increase to 95\% after EHR implementation. Based on these estimates, using a two-sided alpha error of 0.05 and $80 \%$ power, we determined that at least 141 charts would have to be included in each cohort.

The study sample was summarized with descriptive statistics, and chi-squared and generalized linear models were used to examine differences across the cohorts. Multivariable logistical analyses were utilized to determine the effect of the EHR on HIV and PPD completion rates adjusted for parity and insurance type. We also examined the effects of parity and insurance type separately for each cohort year in order to assess year specific effects. All analyses were conducted using SAS statistical software version 9.4 (SAS Institute, Inc., Cary, NC, United States).

\section{Results}

Our overall study population was composed of 647 deliveries. This includes 249 deliveries for which prenatal records were on paper charts in 2011; the EHR cohorts include 208 deliveries in 2012 and 190 deliveries in 2013. Table 1 compares the demographics of the study population. There were no statistically significant differences in age, gestational age at delivery, parity, or insurance type amongst the cohorts.

Adjusted for parity and insurance type, complete PPD and HIV results were statistically less likely to be charted in the 2012 cohort, the year after EHR implementation, compared to the cohort that utilized paper charts (adjusted odds ratio (aOR $0.58(0.37-0.91), \mathrm{p}=0.02)$. The lower rates of complete results after EHR implementation was driven by fewer documented completed PPD tests ( $83.9 \%$ in 2011 vs. $72.6 \%$ in 2012, p=0.003). Completion rates were superior in the 2013 EHR cohort as compared to the 2011 paper chart cohort, however this result is not statistically significant (aOR: $1.40(0.84-2.36), \mathrm{p}=0.20)$.

-Figure 1 shows the PPD and HIV completion rates as stratified by year. Pre-EHR implementation (2011 cohort), both PPD and HIV tests were completed in the $80.7 \%$ of charts. Immediately post-EHR implementation (2012 cohort), both PPD and HIV tests were completed in $72.1 \%$ of charts. Following the refresher course (2013 cohort), 85.7\% of charts had both PPD and HIV tests appropriately documented.

Importantly, only $1.4 \%$ of the 2012 EHR cohort did not have the HIV status documented in the chart, which is a statistically significant improvement from $4.8 \%$ in the 2011 paper chart cohort 
( $\mathrm{p}=0.04$ ). Only $1.1 \%$ of the 2013 EHR cohort did not have HIV status documented, also a statistically significant improvement over the 2011 paper cohort $(\mathrm{p}=0.03)$.

In the year following EHR implementation (2012 cohort), 27.4\% of charts did not have a PPD result documented, which is significantly more than the $16.1 \%$ of paper charts (2011 cohort) that did not have a PPD result documented $(\mathrm{p}=0.003)$. In the second year following EHR implementation (2013 cohort), 13.8\% of charts did not have a PPD result, which is an improvement as compared to the paper chart cohort, but did not reach statistical significance $(\mathrm{p}=0.5)$.

Regardless of whether prenatal care was completed on paper or electronic charts, multiparous women were less likely to have completed both PPD and HIV testing compared to nulliparous women (aOR $0.62(0.45-0.86), \mathrm{p}=0.004)$. This was driven by the cohorts with electronic charts in which multiparous women had significantly lower PPD completion rates than nulliparous women. In 2012, 21.5\% of nulliparas had no PPD status documented, whereas $37.2 \%$ of multiparas had no PPD status documented ( $\mathrm{p}=0.02$ ). In 2013, 7.5\% of nulliparas had no PPD status documented, whereas $21.7 \%$ of multiparas had no PPD status documented $(\mathrm{p}=0.007)$. There was no difference by insurance type in PPD and HIV testing completion rates (aOR $1.21(0.83-1.77), \mathrm{p}=0.33$ )

\section{Discussion}

Our study's mixed results highlight some of the challenges that can accompany EHR implementation. Interestingly, documentation of complete testing of both HIV and PPD decreased following EHR implementation. While documentation of both results improved in the 2013 EHR cohort as compared to the 2011 paper chart cohort, the improvement was not statistically significant.

The finding that documentation of both HIV and PPD results decreased after EHR implementation was driven by decreased rates of PPD documentation, as there was an increased rate of HIV result documentation. This finding underscores some of the inherent strengths and limitations of an EHR. Most laboratory tests-including HIV testing-are automatically uploaded into the EHR; as such, these tests do not require any human intervention to have their results be documented in the EHR. Conversely, PPD test results must be manually entered by the provider interpreting the test. In our study, after EHR implementation, there was a statistically significant improvement in documentation of HIV results. However, PPD result documentation worsened markedly in the first year following EHR implementation, and then improved, but not significantly, in the second year following implementation.

The reason for decreased PPD completion rates is multifactorial. One explanation is a training deficiency: providers may not have learned or remembered how to correctly document the PPD results in the EHR. Another possibility is a lack of post-implementation standardization: certain providers entered PPD results into an outpatient progress note in the EHR but not the prenatal console, which did not autopopulate the PPD result in the patient's inpatient medical record. Interestingly, after a preliminary evaluation of this data following completion of the second cohort, we performed retraining for the providers in an attempt to close knowledge gaps in use of the EHR. While this training resulted in a decreased number of patients without a PPD result documented in the 2013 EHR cohort, this improvement was not statistically significant over the paper charts. Our data suggest providers need multiple educational interventions to ensure accurate EHR use.

It is reassuring that insurance type, used as a proxy for socioeconomic status, did not impact test completion results. That multiparity was found to be associated with a lower completion rate of PPD testing in two of the cohorts may point to the greater demands on time of women caring for another child, making it more challenging for them to return for their test to be interpreted.

A strength of our study is that our study population incorporates cohorts over multiple years within the same institution, allowing us to determine what impact adoption of a comprehensive EHR as well as performance of retraining may have on prenatal test results. The improvement we found in some aspects of prenatal lab completion is consistent with prior studies that have shown EHRs to be superior to paper charts in the prenatal setting. In 2014, Pham-Thomas et al. found that implementation of an electronic prenatal record system increased the availability of prenatal lab results on the labor and delivery unit, and corresponded with a decrease in repeat maternal testing and immunoglobulin administration to newborns [11]. Though not specifically evaluated in our 
study, our results suggest that fewer rapid HIV tests had to be performed on patients upon admission to labor and delivery, as that is our standard when an HIV result is not available at that time.

The study has some limitations. It is a retrospective review, so it is possible that other factors contributed to the difference in charting, despite choosing cohorts that delivered during the same time frame in successive years at the same institution. For example, there was turnover of practitioners doing the charting over the three cohorts, and there was no way to fully standardize training across all practitioners over this timeframe. Because the study was undertaken at a single institution using a single EHR, results may not be generalizable to other settings. We would welcome a study using a different EHR to help determine whether our mixed findings are consistent across different user interfaces, or more inherent to prenatal EHR use in general.

\section{Conclusions}

In conclusion, we found that implementation of a comprehensive EHR in the prenatal setting had mixed results. It improved completion and documentation of HIV results. However, documentation of PPD results worsened following EHR implementation, and improved over time but not in a statistically significant manner. This likely is due in part to the fact that, in many EHRs, provider-entered test results often require multiple steps to achieve correct entry. This is often perceived as more difficult than quickly writing the result on a face sheet in a paper chart. Our findings suggest that, even for discrete results such as a PPD test interpretation, if human intervention is required to enter the result, training and standardization are paramount, but may not be sufficient given that retraining did not improve results significantly. It is possible that a clinical decision support tool, such as an alert that fired if PPD result was not entered by a specified gestational age, could have increased awareness of the lack of documentation. However, our results indicate that the tool would have to include specific instructions on how to enter the result in order to impact documentation completion rates. Our results indicate the importance of ongoing development and continuous improvement of the user interface to increase ease of appropriate documentation of clinician-entered results, vigilance in training and standardization, and appreciation that EHR implementation carries drawbacks as well as benefits. Future study will be necessary to continue quantifying the impact of these various limitations and benefits in terms of healthcare resources, provider experience, and impact on clinical outcomes.

\section{Clinical Relevance Statement}

Implementation of an EHR does not ensure that all prenatal lab results will be documented more completely than on paper charts. Test result characteristics and provider training and standardization must be taken into account.

\section{Conflicts of Interest}

The authors report no conflict of interest. There was no source of financial support for this study, nor funding from any organization.

\section{Human and Animal Subjects}

There were no human or animal subjects included in this project.

\section{Acknowledgement}

We wish to acknowledge Sanae Nakagawa, University of California, San Francisco, for her assistance with statistical analysis.

Results from the 2011 and 2012 cohorts were presented as a poster at the 62nd ACOG Clinical Meeting, April 26-30, 2014, Chicago, Illinois. 


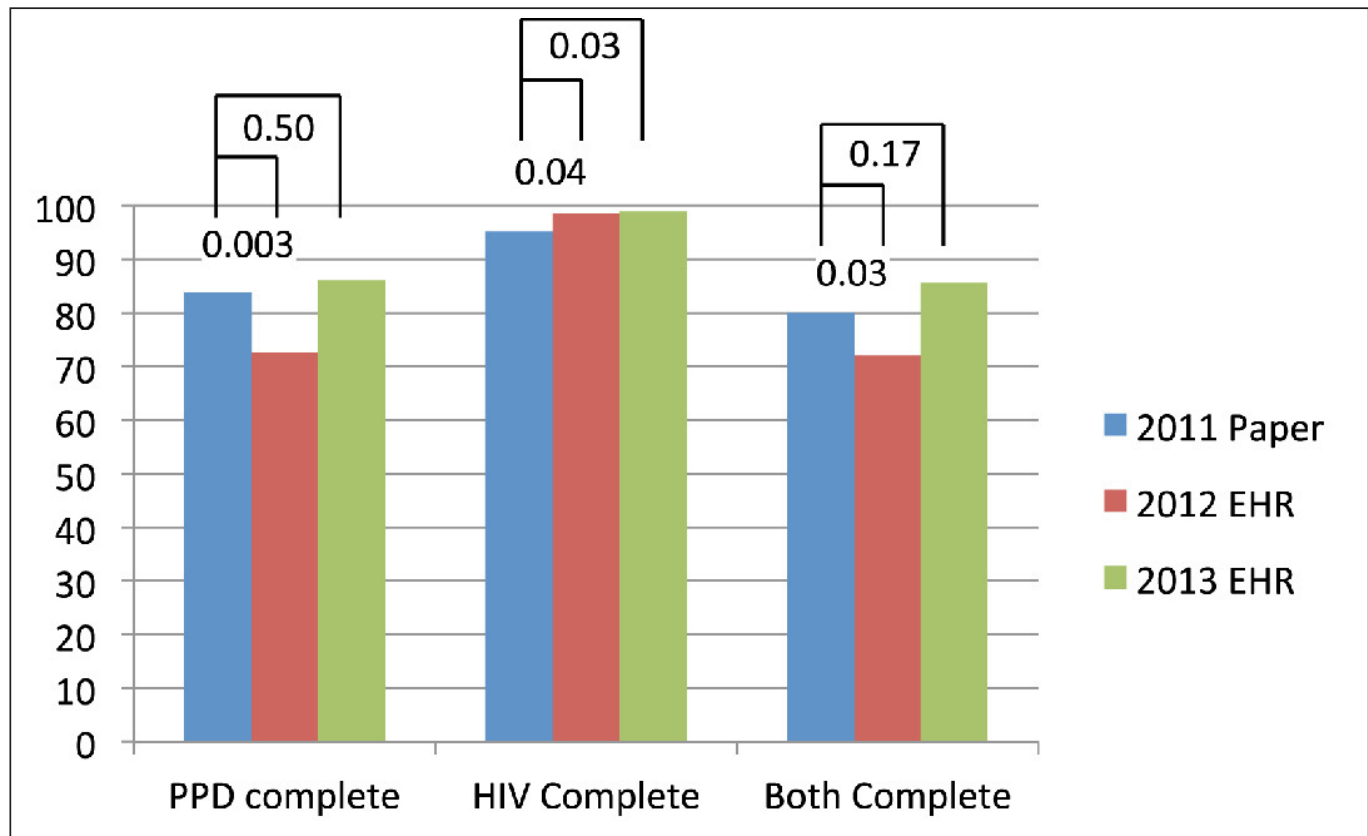

Fig. 1 Completion rates of documented purified protein derivative (PPD) and Human Immunodeficiency Virus (HIV) results in the 2011 paper chart cohort (blue); the 2012 electronic health record cohort (red); and the 2013 electronic health record cohort, after retraining (green). 
Table 1 Demographics of the Study population $(n=647)$

\begin{tabular}{|c|c|c|c|c|}
\hline Parameter & $\begin{array}{l}2011(n=249) \\
\text { Paper chart }\end{array}$ & $\begin{array}{l}2012(n=208) \\
\text { EHR }\end{array}$ & $\begin{array}{l}2013(n=190) \\
\text { EHR }\end{array}$ & $P$ \\
\hline Age (years) & $32.7 \pm 5.9$ & $31.9 \pm 5.5$ & $32.6 \pm 5.7$ & 0.309 \\
\hline Gestational age at delivery (weeks) & $39.4 \pm 1.2$ & $39.4 \pm 1.1$ & $39.3 \pm 1.1$ & 0.747 \\
\hline \multicolumn{4}{|l|}{ Parity } & 0.179 \\
\hline Nulliparous & $135(54.2 \%)$ & $130(62.5 \%)$ & $106(55.8 \%)$ & \\
\hline Multiparous & $114(45.8 \%)$ & $78(37.5 \%)$ & $84(44.2 \%)$ & \\
\hline \multicolumn{4}{|l|}{ Insurance status } & 0.191 \\
\hline Private & $186(74.7 \%)$ & $143(68.8 \%)$ & $128(67.4 \%)$ & \\
\hline Public & $63(25.3 \%)$ & $65(31.3 \%)$ & $62(32.6 \%)$ & \\
\hline
\end{tabular}

Data are $\mathrm{n}(\%)$ or mean \pm standard deviation unless otherwise noted 


\section{References}

1. Blumenthal D. Stimulating the adoption of health information technology. New Engl J Med 2009; 360(15): 1477-1479.

2. Metz JP, Son SJ, Winter RO, Chae S. Increasing timely and available prenatal studies by electronic health records. J Am Board Fam Med 2011; 24(4): 344-350.

3. Persell SD, Kaiser D, Dolan NC, Andrews B, Levi S, Khandekar J, Gavagan T, Thompson JA, Friesema EM, Baker DW. Changes in performance after implementation of a multifaceted electronic-health-record based quality improvement system. Med Care 2011; 49(2): 117-125.

4. Varroud-Vial, M. Improving diabetes management with electronic medical records. Diabetes Metab 2011; 37(Suppl 4): S48-S52.

5. Eden KB, Messina R, Li H, Osterweil P, Henderson CR, Guise JM. Examining the value of electronic health records on labor and delivery. Am J Obstet Gynecol 2008; 199(3): 307. e1-e9.

6. Poon EG, Wright A, Simon SR, Jenter CA, Kaushal R, Volk LA. Relationship between use of electronic health record features and health care quality: results of a statewide survey. Med Care 2010;48(3): 203-209.

7. Samaan ZM, Klein MD, Mansour ME, DeWitt TG. The impact of the electronic health record on an academic pediatric primary care center. J Ambul Care Manage 2009; 32(3): 180-187.

8. Jha AK, DesRoches CM, Campbell EG, Donelan K, Rao SR, Ferris TG, Shields A, Rosenbaum S, Blumenthal D. Use of electronic health records in U.S. Hospitals. New Engl J Med 2009; 360(16): 1628-1638.

9. American Academy of Pediatrics \& The American Congress of Obstetricians \& Gynecologists. Guidelines for Perinatal Care: 7th Edition. Elk Grove Village (IL), Washington (DC): AAP, American College of Obstetricians and Gynecologists 2012.

10.Bernstein PS, Farinelli C, Merkatz IR. Using an electronic medical record to improve communication within a prenatal care network. Obstet Gynecol 2005; 105(3): 607-612.

11.Pham-Thomas N, Pereira N, Powel AM, Croft DJ, Guilfoil DS, and Montgomery OC. Outcomes of Effective Transmission of Electronic Prenatal Records from the Office to the Hospital. Obstet Gynecol 2014; 124(2): 317-322.

12. Communicable Disease Control and Prevention, San Francisco Department of Public Health. Tuberculosis in San Francisco, 2014. http://sfcdcp.org/document.html?id=974. 